\title{
Automatic Modulation Classification for Cognitive Radios using Cumulants based on Fractional Lower Order Statistics
}

\author{
M. Narendar ${ }^{1}, \underline{\text { A. P. Vinod }}{ }^{1}$, A.S. Madhukumar ${ }^{1}$, and Anoop Kumar Krishna ${ }^{2}$ \\ ${ }^{1}$ School of Computer Engineering, Nanyang Technological University, Nanyang Avenue, Singapore. \\ ${ }^{2}$ EADS Innovation Works, Seletar Aerospace View ,Singapore. anoop.krishna@eads.net \\ ${ }^{1}$ Email: \{nare0003, asvinod, asmadhukumar\}@ntu.edu.sg.
}

\begin{abstract}
Automatic modulation classification (AMC) finds various applications in cognitive radios. This paper presents a method for the automatic classification using cumulants derived using fractional lower order statistics. The performance of the classifier is presented in the form of probability of correct classification under noisy and fading conditions. Unlike many of the conventional methods, the proposed method does not require a priori knowledge of signal parameters. The proposed method is also more robust to different noises. Simulation results show that the proposed method can achieve better classification accuracy when compared to conventional cumulant based AMC method, in various impulsive noise conditions.
\end{abstract}

\section{Introduction}

Automatic modulation classification (AMC) is a method to automatically determine the modulation type of the transmitted signal by performing one or many signal processing techniques on the received signal. Blind classification of the received signal finds an important application in software defined radio (SDR) and cognitive radio (CR) systems. An ideal CR must be able to detect/sense the signals present in the spectrum and classify them automatically for the efficient usage of spectral resources [1]. The challenging task in AMC is to classify the received signal into a modulation type without any or little priori information about the transmitted signal. This becomes even more difficult when the received signal is corrupted by adverse channel conditions such as noise and fading. AMC is broadly classified into two major methods- decision-theoretic methods and pattern recognition methods.

Decision-theoretic approach is generally likelihood based detectors (LBD) [2], whereas pattern recognition methods are feature based detectors (FBD) [3]. In LBD, appropriate likelihood ratio functions of the received signal is generated based on the multiple hypotheses test and the decision is made by comparing the likelihood ratio against a pre-determined threshold. LBD is optimal but computationally complex. On the other hand, in the FBD approach, the AMC extracts key features necessary for classification from the received signal and determines the modulation type of the transmitted signal based on those features extracted, using a decision rule. Existing methods have used cumulants, based on higher-order statistics (HOS) up to eighth-order [3]. FBD based methods are non-optimal but very easy to implement and can achieve high probability of correct classification $\left(P_{c c}\right)$. LBD are not robust in the presence of phase or frequency offsets, residual channel effects or other fading effects. It also assumes noise to be Gaussian while calculating the likelihood functions [2]. However, in real communication scenario, it is sometimes inappropriate to model the noise as just purely Gaussian. Most of the papers in the literature address the above mentioned issues by applying techniques such as approximation, and/or estimation [4]. In this paper, an FBD method for AMC in the presence of noise and fading is proposed. A fractional lower order statistics (FLOS) based fourth-order cumulants acts as the key feature for classification. The FLOS based analysis, allows noise to be more generic and handles interference robustly [6]. The decision rule to determine $P_{c c}$ is based on a likelihood ratio test which gives good classification performance. The major advantage of the proposed method is its robustness in different noise and fading conditions The present paper is organized a follows: In Section 2, the classification problem is outlined based on the classical decision theory. In Section 3, the proposed method for AMC based on FLOS and cumulants are discussed in detail. The thresholds necessary for the implementation of the method are introduced. Section 4 reviews the proposed method and its performance based on the probability of classification in various impulsive noise conditions and fading. Finally, Section 5 concludes the paper. 


\section{Problem Definition}

We consider the propagation of symbols over a channel which might have Gaussian or other impulsive noises added to the symbols which are faded. This can be described as

$$
r(n)=\sum_{k=0}^{L-1} h(k) s(n-k)+w(n)
$$

where $r(n)$ is the received symbol sequence whose modulation type and symbol energy are unknown. $s(n)$ is the transmitted symbol which may be obtained from one of the $M$ possible modulation schemes, whose constellations are obtained under same average power condition. $h(n)$ is the channel coefficients corresponding to a multipath channel of length $L$ and $w(n)$ is the additive noise. Based on the classification theory, there are $M$ modulations to be classified, which are denoted as $m_{1}, m_{2}, \ldots . . m_{M}$. The probability of correct classification is formulated as

$$
P_{c c}=\sum_{i=1}^{M} P\left(m_{i} \mid m_{i}\right) P_{m_{i}}
$$

where $P_{m_{i}}$ is the probability of modulation type $m_{i}$ and $P\left(m_{i} \mid m_{i}\right)$ is the conditional probability of an occurrence of a modulation when it is actually transmitted. Since all modulation types are equally likely, $P_{m_{i}}$ is equal to $1 / M$. In order to make the classifier robust to various noisy conditions, it is designed for processes that are a generalization of Gaussian processes. For example, literature shows that, impulsive noises can be better modeled as stationary symmetric alpha stable $(S \alpha S)$ processes [5]. Gaussian noises are in fact a special class of the larger class of stable distributions with the characteristic exponent, $\alpha=2$. It is shown in [6] that, for a complex $S \alpha S$ random variable $X$, it has a finite fractional lower order moments (FLOM) for $q<\alpha \leq 2$ and its fractional, $q^{\text {th }}$ order correlation sequence can be defined as

$$
\left\langle X_{n}, X_{m}\right\rangle_{p}=E\left(X_{n} \cdot\left(X_{m}\right)^{q-1}\right)
$$

where $E(\quad)$ is the statistical expectation of the random variables. It can be seen that, for $q=2$, (3) gives autocorrelation sequence of the random variable $X$. The FLOS based algorithms have been proved to be robust for impulsive, as well as Gaussian data in the literature. In this paper, we adapt the idea of FLOS into cumulants which are used to perform modulation classifications described in the following section.

\section{Proposed Automatic Modulation Classification Method}

A pattern recognition based approach is adopted for the proposed AMC classification. Though the proposed method is based on fourth order cumulants as a key feature for classification, a modification to the original fourth order cumulants is done using FLOS to make it more robust to various noise conditions. A typical second and fourth order cumulants are given by the following relations respectively: [3, 4]

$$
\begin{gathered}
C_{21}(s)=\operatorname{cum}\left[s, s^{*}\right]=E\left(|s|^{2}\right) \\
C_{42}(s)=\operatorname{cum}\left[s, s, s^{*}, s^{*}\right]=E\left(|s|^{4}\right)-\left|E\left(s^{2}\right)\right|^{2}-2 E^{2}\left(|s|^{2}\right)
\end{gathered}
$$

In order to make the cumulant based classifier [4] robust to different noises, FLOS can be incorporated into the cumulant expression in two ways as shown in (6) and (8)

$$
C_{42 p}(s)=\operatorname{cum}\left[s^{p}, s^{1-p}, s^{* p}, s^{* 1-p}\right]
$$

where $0 \leq p \leq \alpha / 2$. In case of Gaussian noise, $\alpha$ is 2 , and hence $p$ takes values between 0 and 1 . This is normalized as

$$
\hat{C}_{42 p}(s)=\frac{C_{42 p}(s)}{\left(C_{21}(s)\right)^{2 p}}
$$




$$
C_{42}(s)_{F L O S}=E\left(|s|^{4 p}\right)-\left|E\left(s^{2}\right)\right|^{2 p}-2 E^{2}\left(|s|^{2 p}\right)
$$

Now, by expanding the cumulant expression and then incorporating FLOS, we can obtain a better expression for the classifier as shown below

$$
\hat{C}_{n 42 p}(s)=\frac{C_{42}(s)_{F L O S}}{\left\{C_{21}(s)_{F L O S}\right\}^{2}}=\frac{\frac{\sum_{n=1}^{N}|s(n)|^{4 p}}{N}-\frac{\left|\sum_{n=1}^{N} s^{2}(n)\right|^{2 p}}{N^{2}}-2\left\{\frac{\sum_{n=1}^{N}|s(n)|^{2 p}}{N}\right\}^{2}}{\left\{\frac{\sum_{n=1}^{N}|s(n)|^{2 p}}{N}\right\}^{2}}
$$

It can be seen that the cumulant method in [4] is a special case of the proposed method for $p=1$. The theoretical values of normalized cumulants based on FLOS ((6) and (9)) are calculated for various values of $p$ for different modulations and plotted in Fig. 1(a) and Fig. 1(b) respectively. Therefore, for a set of $M$ modulations, each modulation has a unique normalized cumulant value based on FLOS. Fig. 1(b) shows that, for $p=1$, the proposed method approaches the theoretical fourth order cumulant values for all modulations as given in [4]. Thus, the proposed method is a generalized classsifier, robust for different types of noise scenarios. It estimates the normalized cumulants of the received symbols and compares it with the theoretical thresholds to determine the modulation type. The performance evaluation and working of the AMC method is presented in the next section with a typical example.

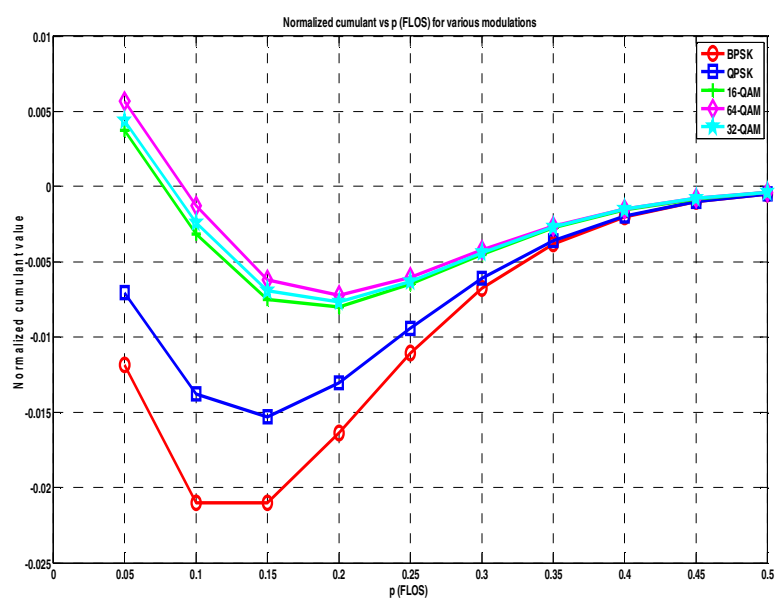

Figure. 1(a). Normalized theoretical cumulant value $\hat{C}_{42 p}(s)$ vs $p$.

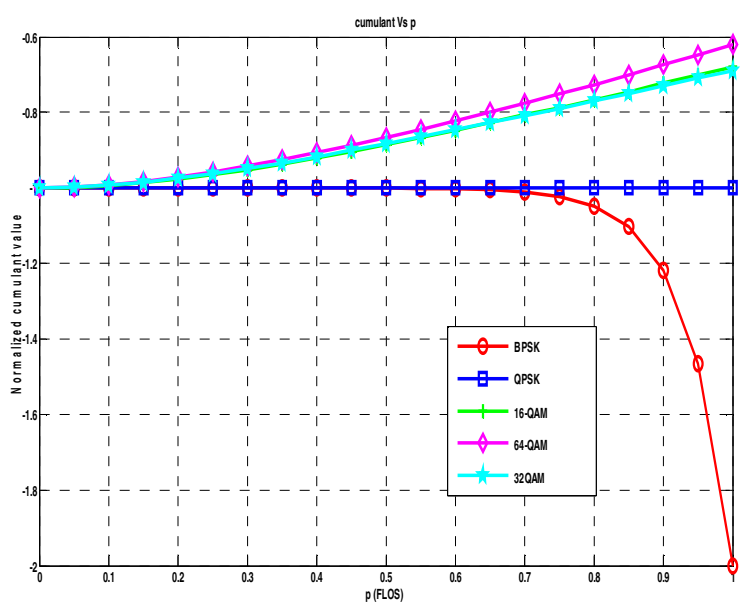

Figure. 1(b). Normalized theoretical cumulant value $\hat{C}_{n 42 p}(s)$ vs $p$.

\section{Performance Evaluation and Simulations}

In this section, the proposed AMC method is evaluated using Monte Carlo simulations in terms of the probability of correct classification $\left(P_{c c}\right)$, as explained in Section 2. In order to validate the algorithm, a modulation candidate set of $\{$ BPSK, QPSK, 16-QAM, 64-QAM $\}$ is taken. $P_{c c}$ is given by

$$
P_{c c}=\frac{1}{4}(P(B P S K \mid B P S K)+P(Q P S K \mid Q P S K)+P(16 Q A M \mid 16 Q A M)+P(64 Q A M \mid 64 Q A M))
$$

The propagation environment is assumed as multipath Rayleigh faded channel where number of paths $(L)$ is fixed as 4. The channel coefficients are uniformly distributed within the given delay spread. Noise is modeled as a $S \alpha S$ process with a known measure of dispersion $\left(\partial_{g}\right)$. Monte Carlo simulations are done to test the performance of the proposed AMC method with 2048 symbols collected in each trial. The estimate of the normalized cumulants of the received samples are calculated for the known noise condition as $\hat{C}_{n 42 p}(s)=\frac{C_{42}(s)_{F L O S}}{\left\{C_{21}(s)_{F L O S}-\partial_{g}^{2 p}\right\}^{2}} \cdot p$ is chosen as 0.9.

The estimate is compared with the theoretical thresholds based on Fig. 1(b). A simple likelihood ratio test is employed by calculating the minimum distance between the estimated and the theoretical values. The SNR vs $P_{c c}$ is plotted for highly and slightly impulsive noises in fading for the proposed and conventional method using cumulants 
[4] in Fig. 2(a) and Fig. 2(b) respectively. The proposed method outperforms the method in [4] in the case of a highly impulsive noise and is slightly better for slightly impulsive noise (which is more closer to AWGN). However, when the classifier approaches the method in [4] corresponding to $p=1$ for AWGN case, the performance of the proposed method is identical to that of [4]. Thus, it is shown using these plots that, the proposed method is robust to different noise scenarios.

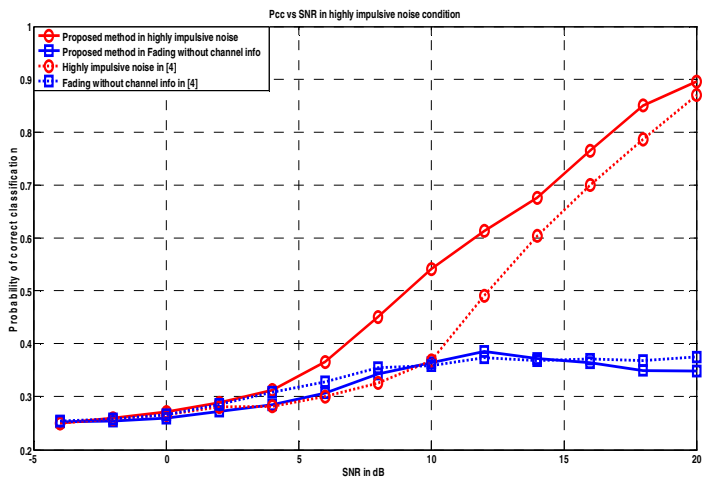

Figure. 2(a). Comparison of $P_{c c}$ vs SNR in highly impulsive noise.

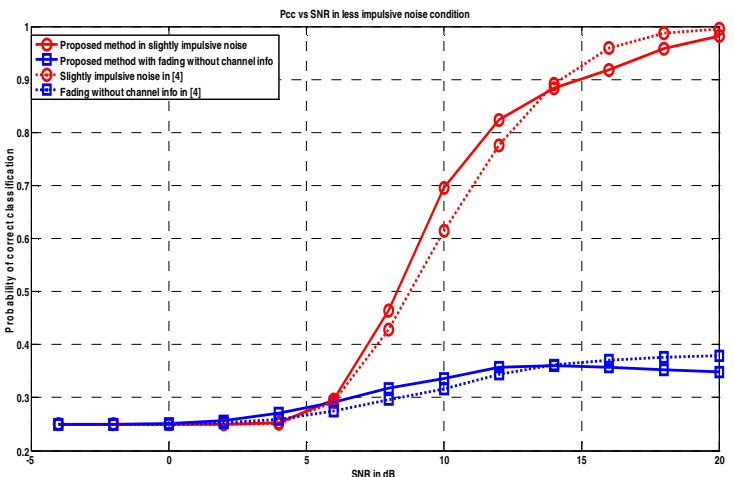

Figure. 2(b). Comparison of $P_{c c}$ vs SNR in slightly impulsive noise.

\section{Conclusions}

In this paper, a new pattern recognition based automatic modulation classification algorithm, based on FLOS and cumulants is proposed. The challenge in designing a classifier is the lack of prior knowledge about the signal and channel information. Relevant features required for AMC are formulated using FLOS based cumulants, which are then compared with theoretical thresholds to perform classification. Monte Carlo simulations show that the proposed algorithm gives significant AMC performance, when compared with the conventional cumulant based AMC in impulsive noises. The proposed method based on FLOS, allows noise to be generic, and approaches the conventional method based on cumulants when the noise is purely Gaussian. The challenge lies in improving the classification probability for lower SNRs in impulsive noises and in the presence of multipath fading, which would be the focus of our future work.

\section{Acknowledgments}

This work was supported by European Aeronautic Defense and Space (EADS) Singapore Pte Ltd., Singapore.

\section{References}

1. J. Mitola and G. Q., Maguire "Cognitive radio: making software radios more personal," IEEE Personal Commun. Mag., vol. 6, no. 4, pp. 13-18, Aug. 1999.

2. C.-Y. Huan and A. Polydoros, "Likelihood methods for MPSK modulation classification," IEEE Trans. Commun., vol. 43, no. 2/3/4, pp. 1493-1504, Feb./Mar./Apr. 1995.

3. O. A. Dobre, Y. Bar-Ness, and W. Su, "Higher-order cyclic cumulants for high order modulation classification," in Proc. IEEE MILCOM, vol. 1, Oct. 2003, pp. 112-117.

4. Wu, H.-C., Saquib, M., and Yun, Z.: 'Novel automatic modulation classification using cumulant features for communications via multipath channels', IEEE Trans. Wirel. Commun., 2008, 7, (8), pp. 3098-3105

5. M. Shao and C. L. Nikias, Signal Processing With Alpha-Stable Distributions and Applications. New York: Wiley, 1995.

6. M. Rupi, P. Tsakalides, E. D. Re, C. L. Nikias. Constant modulus blind equalization based on fractional lowerorder statistics. Signal Processing, 2004, 88(5):881-894. 